\title{
Publisher Correction: Autophagy promotes growth of tumors with high mutational burden by inhibiting a T-cell immune response
}

Laura Poillet-Perez, Daniel W. Sharp, Yang Yang (1), Saurabh V. Laddha (D, Maria Ibrahim, Praveen K. Bommareddy (D), Zhixian Sherrie Hu, Joshua Vieth, Michael Haas, Marcus W. Bosenberg, Joshua D. Rabinowitz (D), Jian Cao(D), Jun-Lin Guan $\mathbb{D}$, Shridar Ganesan, Chang S. Chan, Janice M. Mehnert, Edmund C. Lattime $\mathbb{B}$ and Eileen White $\mathbb{B}$

Correction to: Nature Cancer https://doi.org/10.1038/s43018-020-00110-7, published online 18 September 2020.

In the version of this article initially published, Supplementary Tables 1-3 and 11-13 were omitted from the Supplementary Information file. The error has been corrected.

\section{Additional information}

Supplementary information The online version contains supplementary material available at https://doi.org/10.1038/s43018-021-00252-2.

Published online: 16 August 2021

https://doi.org/10.1038/s43018-021-00252-2

(C) The Author(s), under exclusive licence to Springer Nature America, Inc. 2021 